\title{
The Effects of Partial Observability in SLAM
}

\author{
Juan Andrade-Cetto and Alberto Sanfeliu \\ Institut de Robòtica i Informàtica Industrial, UPC-CSIC \\ Llorens Artigas 4-6, 08028 Barcelona, Spain \\ \{cetto, sanfeliu\}@iri.upc.es
}

\begin{abstract}
In this article, we show that partial observability hinders full reconstructibility of the state space in SLAM, making the final map estimate dependent on the initial observations, and not guaranteeing convergence to a positive semi-definite covariance matrix. By characterizing the form of the total Fisher information we are able to determine the unobservable state space directions. To overcome this problem, we formulate new fully observable measurement models that make SLAM stable.
\end{abstract}

\section{INTRODUCTION}

The study of stochastic models for Simultaneous Localization and Map Building (SLAM) in mobile robotics has been an active research topic for over fifteen years. Within the Kalman filter (KF) approach to SLAM, seminal work by Smith and Cheeseman [1] suggested that as successive landmark observations take place, the correlation between the estimates of the location of such landmarks in a map grows continuously. This observation was ratified by Dissanayake et al. [2] with a proof showing that the estimated map converges monotonically to a relative map with zero uncertainty. They also showed how the absolute accuracy of the map reaches a lower bound defined only by the initial vehicle uncertainty, and proved it for a one-landmark vehicle with no process noise.

In this communication we address these results as a consequence of partial observability. We show that full reconstruction of the map state vector is not possible with typical measurement models, regardless of the vehicle model chosen, and propose new fully observable models. Also, we show experimentally how the expected error in state estimation is proportional to the number of landmarks used.

An explicit solution to the SLAM problem for a onedimensional vehicle called the monobot was presented by Gibbens et al. [3]. It shed some light on the relation between the total number of landmarks and the asymptotic values for the state error covariance $\mathbf{P}$. They observed for example, that in SLAM, the rate of convergence of $\mathbf{P}$ is fixed, and that its asymptotic value is independent of the plant variance. In their solution to the 1-d Brownian motion case, the state error covariance is linked to the total number of landmarks in the form of the total Fisher information $I_{T}=\sum_{1}^{n}\left(1 / \sigma_{w}^{2}\right)$. The expression indicates the "informational equivalence of the measurements and the innovations" [4], and was derived from a simple likelihood function, one that does not contain the fully correlated characteristics of the measurement model. We derive a more general expression for the total Fisher

This work was supported by the Spanish Council of Science and Technology under project DPI 2001-2223.

IEEE International Conference on Robotics and Automation New Orleans. April 2004. pD. 397-402 information in SLAM that shows explicitly the unobservable directions of the state space.

In summary; in SLAM, the state space constructed by appending the robot pose and the landmark locations is fully correlated, a situation that hinders full observability. Moreover, the modelling of map states as static landmarks yields a partially controllable state vector. The identification of the first of these problems, and the steps taken to palliate it, are covered in this article. The effects of partial controllability in SLAM are covered in [5].

The paper is structured as follows. In Section II we analyze the steady state behavior of the error state covariance in SLAM for the monobot, and show how the steady state of the filter will always depend on the initial noise parameters. The effect is known as marginal stability [6], and is in general an undesirable feature in state estimation. In Section III we derive an expression for the total Fisher information in SLAM. The analysis yields a closed form solution that shows, explicitly, the unobservable directions of the map state.

Marginal filter stability and the singularity of the Fisher information matrix are equivalently consequences of having partial observability. Section IV is devoted to the computation of general expressions for the bases of the controllable and observable subspaces in SLAM. These expressions are later simplified in Sections V and VI for the monobot, and for a planar wheeled vehicle. We prove, in the end, that the angle between these two subspaces is determined only by the total number of landmarks in the map. The result is that as the number of landmarks increases, the state components get closer to being reconstructible.

In Section VII we show how partial observability in SLAM can be avoided by adding a fixed external sensor to the state model, or equivalently, by setting a fixed landmark in the environment to serve as global localization reference. Full observability yields the existence of a (not necessarily unique) steady state positive semi-definite solution for the error covariance matrix, guaranteeing a steady flow of the information about each state component, and preventing the uncertainty (error state covariance) from becoming unbounded [4].

\section{Steady State Behavior OF KF-SLAM}

We start the discussion with a pictorial representation of the asymptotic behavior of the KF-SLAM algorithm. The steady state covariance matrix is given by the solution of the Ricatti 
equation

$$
\mathbf{P}=\mathbf{F}\left(\mathbf{P}-\mathbf{P H}^{\top}\left(\mathbf{H} \mathbf{P} \mathbf{H}^{\top}+\mathbf{W}\right)^{-1} \mathbf{H P}\right) \mathbf{F}^{\top}+\mathbf{V}
$$

with $\mathbf{F}$ and $\mathbf{H}$ the plant and measurement model Jacobians, respectively; and $\mathbf{V}$ and $\mathbf{W}$ the motion and sensor noise covariances.

For the linear fully observable case, the solution to the Ricatti equation will converge to a steady state covariance only if the pair $\{\mathbf{F}, \mathbf{H}\}$ is completely observable. If in addition, the pair $\{\mathbf{F}, \mathbf{I}\}$ is completely controllable, then the steady state covariance is a unique positive definite matrix, independent of the initial covariance $\mathbf{P}_{0 \mid 0}$ [4]. These two conditions are not satisfied in general in SLAM, and for the linear case, the solution of (1) is a function of the initial vehicle pose covariance $\mathbf{P}_{r, 0 \mid 0}, \mathbf{V}, \mathbf{W}$, and the total number of landmarks $n$. Note however that, for the nonlinear case, the computation of the Jacobians $\mathbf{F}$ and $\mathbf{H}$ will in general also depend on the steady state value of $\mathbf{x}$.

Consider a linear one-dimensional vehicle, i.e., a monobot. The evolution of the error covariance matrix is independent of the state input, and measurements throughout the run of the algorithm. For a monobot with perfect data association and constant motion and sensor uncertainty, the computation of the Kalman gain could even be performed offline. That is, the asymptotic (steady state) behavior of the filter, and its rate of convergence are always the same, regardless of the actual motions and measurements.

Fig. 1 shows the steady state vehicle and landmark variances of the KF-SLAM algorithm applied to a monobot when observations of 1, 2, 3, and 50 landmarks are available. The figure plots the influence of each of the noise variances $\mathbf{V}$ and $\mathbf{W}$ with respect to the final vehicle and landmark uncertainty.

All final state estimates are bounded by below by the initial vehicle variance $\mathbf{P}_{r, 0 \mid 0}=1$. Meaning one can never estimate the vehicle and landmark locations with more accuracy than what was available at the first sighting [2], but certainly can do worse; that being dictated by the values of $\mathbf{V}, \mathbf{W}$, and the total number of landmarks $n$.

\section{TOTAL FISHER INFORMATION}

Under the Gaussian assumption for the vehicle and sensor noises, the Kalman filter is the optimal minimum mean square error estimator. And, as pointed out in [4], minimizing the least squares criteria $E\left[\tilde{\mathbf{x}}_{k+1 \mid k+1} \tilde{\mathbf{x}}_{k+1 \mid k+1}^{\top}\right]$, is equivalent to the maximization of a likelihood function $\Lambda(\mathbf{x})$ given the set of observations $Z^{k}$; that is, the maximization of the joint probability density function of the entire history of observations, $\Lambda(\mathbf{x})=\prod_{i=1}^{k} p\left(\mathbf{z}_{i} \mid Z^{i-1}\right)$, where $\mathbf{x}$ is the augmented map state (vehicle and landmark estimates), and $\mathbf{z}_{i}$ the entire observation vector at time $i$.

Given that the above pdfs are Gaussian, and that $E\left[\mathbf{z}_{i}\right]=$ $\mathbf{H} \mathbf{x}_{i \mid i-1}$, the pdf for each measurement in SLAM is $p\left(\mathbf{z}_{i} \mid Z^{i-1}\right)=N\left(\tilde{\mathbf{z}}_{i \mid i-1} ; \mathbf{0}, \mathbf{S}_{i}\right)$, with $\mathbf{S}_{i}=E\left[\tilde{\mathbf{z}}_{i \mid i-1} \tilde{\mathbf{z}}_{i \mid i-1}^{\top}\right]$.

In practice however, it is more convenient to consider the $\log$ likelihood function $\ln \Lambda(\mathbf{x})$. The maximum of $\ln \Lambda(\mathbf{x})$ is at the value of the state $\mathbf{x}$ that most likely gave rise to the

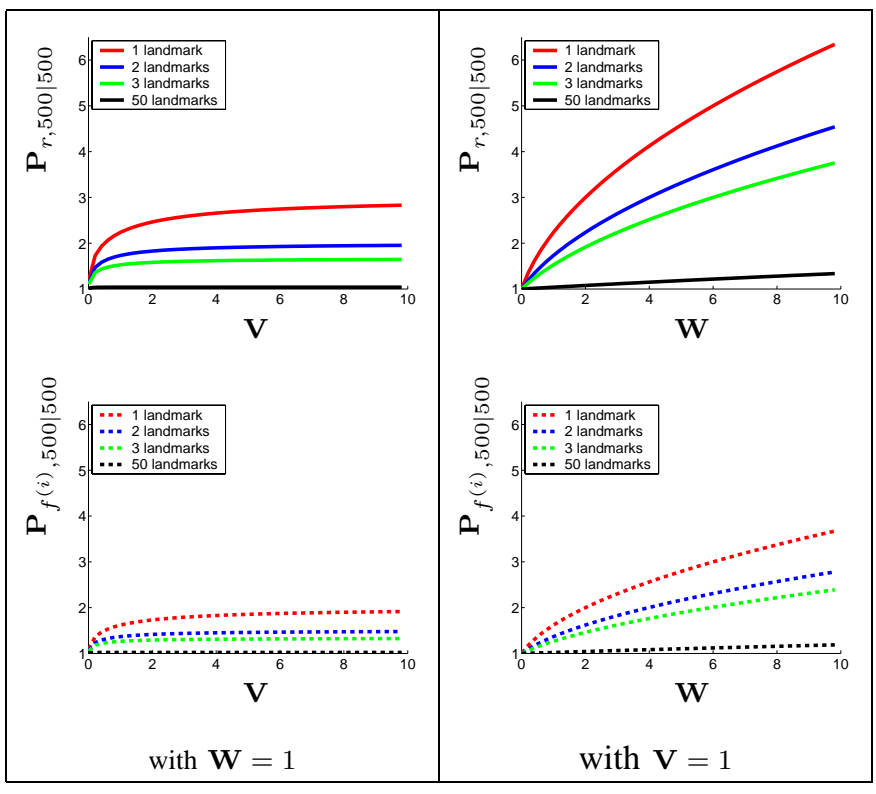

Fig. 1. Final vehicle and landmark localization variances after 500 iterations of SLAM for a monobot with initial localization variance $\mathbf{P}_{r, 0 \mid 0}=1$, and various values for the plant and sensor noise variances.

observed data $Z^{k}$, and is obtained by setting its derivative with respect to $\mathbf{x}$ equal to zero, which gives

$$
\nabla_{\mathbf{x}} \ln \Lambda(\mathbf{x})=\sum_{i=1}^{k} \mathbf{H}^{\top} \mathbf{S}_{i}^{-1} \tilde{\mathbf{z}}_{i \mid i-1}
$$

An intuitive interpretation of the maximum of the loglikelihood is that the best estimate for the state $\mathbf{x}$, in the least squares sense, is the one that makes the sum of the entire set of Mahalanobis distances $\sum_{i=1}^{k} \tilde{\mathbf{z}}_{i \mid i-1}^{\top} \mathbf{S}_{i}^{-1} \tilde{\mathbf{z}}_{i \mid i-1}$ as small as possible. A measure that is consistent with the spatial compatibility test described in [7].

The Fisher information matrix, a quantification of the maximum existing information in the observations about the state $\mathbf{x}$, is defined in [4] as the expectation on the dyad of the gradient of $\ln \Lambda(\mathbf{x})$, that is, $\mathbf{J}=E\left[\left(\nabla_{\mathbf{x}} \ln \Lambda(\mathbf{x})\right)\left(\nabla_{\mathbf{x}} \ln \Lambda(\mathbf{x})\right)^{\top}\right]$. Taking the expectation on the innovation error in the above formula gives the sum

$$
\mathbf{J}=\sum_{i=1}^{k} \mathbf{H}^{\top}\left(\mathbf{H} \mathbf{P} \mathbf{H}^{\top}+\mathbf{W}\right)^{-1} \mathbf{H}
$$

It is easy to verify that in the linear case, this expression for the total Fisher information is only a function of $\mathbf{P}_{r, 0 \mid 0}, \mathbf{V}$, and $\mathbf{W}$. If, on the other hand, the EKF is used, the Jacobian $\mathbf{H}$ in (3) should be evaluated at the true value of the states $\mathbf{x}_{0}, \ldots \mathbf{x}_{k}$. Since these are not available, an approximation is obtained at the estimates $\mathbf{x}_{i \mid i-1}$. The pre and post multiplying $\mathbf{H}$ is, in this context, also known as the sensitivity matrix.

A necessary condition for the estimator (the Kalman filter) to be consistent in the mean square sense is that there must be an increasing amount of information about the state $\mathbf{x}$ in the measurements. That is, as $k \rightarrow \infty$, the Fisher information must 


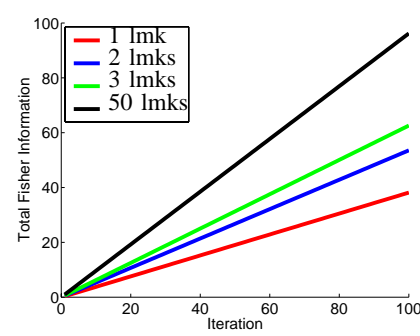

Fig. 2. First entry in the total Fisher information matrix $\left(\sum \sum \varsigma_{i j}\right)$ for a monobot with variance parameters $\mathbf{P}_{r, 0 \mid 0}=\mathbf{V}=\mathbf{W}=1$, and various sizes for the measurement vector.

also tend to infinity. Fig. 2 shows this for the monobot with constant parameters $\mathbf{P}_{r, 0 \mid 0}=\mathbf{V}=\mathbf{W}=1$, and various sizes for the observation vector. Notice how, as the total number of landmarks grows, the total Fisher information also grows, directly relating the number of landmarks to the amount of information available for state estimation in SLAM.

Solving for the $k$-th sum term in $\mathbf{J}$ for the monobot,

$$
\mathbf{J}_{k}=\left[\begin{array}{cc}
\sum \sum \varsigma_{i j} & -\varsigma \\
-\boldsymbol{\varsigma}^{\top} & \mathbf{S}_{k}^{-1}
\end{array}\right]
$$

with $\varsigma_{i j}$ the $i j$-th entry in $\mathbf{S}_{k}^{-1}$, and $\boldsymbol{\varsigma}=\left[\sum \varsigma_{1 i}, \ldots, \sum \varsigma_{n i}\right]$.

Citing Bar-Shalom et al. [4]: "a singular Fisher information matrix means total uncertainty in a subspace of the state space, that is, the information is insufficient for the estimation problem at hand." Unfortunately, it can be easily shown, at least for the monobot case, that the first row (or column) of $\mathbf{J}$ is equivalent to the sum of the rest of the rows (or columns), producing a singular total Fisher information matrix. Thus, SLAM is unobservable.

This is a consequence of the form of the Jacobian $\mathbf{H}$, i.e, of the full correlation in SLAM. Zero eigenvalues of $\mathbf{H}^{\top} \mathbf{S}^{-1} \mathbf{H}$ are an indicator of partial observability, and the corresponding vectors give the unobservable directions in state space.

So for example, for a one-landmark monobot, the innovation covariance is the scalar $s=\sigma_{r}^{2}-2 \rho_{r f} \sigma_{r} \sigma_{f}+\sigma_{f}^{2}+\sigma_{w}^{2}$, and since $\mathbf{H}=[-1,1]$, the Fisher information matrix in (3) evaluates to

$$
\mathbf{J}=\left[\begin{array}{rr}
1 & -1 \\
-1 & 1
\end{array}\right] \sum_{i=1}^{k} \frac{1}{s_{i}}
$$

The unobservable direction of the state space is the eigenvector associated to the null eigenvalue of $\mathbf{J}$, we denote it for now $\mathbf{E}_{\text {KerR }}$ (the name will be clear soon), and evaluates to

$$
\mathbf{E}_{\mathrm{KerR}}=\left(\begin{array}{l}
1 \\
1
\end{array}\right)
$$

\section{ObSeRVABle Subspace}

To see what part of the state space is compromised by full correlation, we now develop closed form expressions for the bases of the observable and controllable subspaces in SLAM and relate them to the total number of landmarks used.

The linearized state model is

$$
\begin{aligned}
\mathbf{x}_{k+1} & =\mathbf{F} \mathbf{x}_{k}+\mathbf{v}_{k} \\
\mathbf{z}_{k+1} & =\mathbf{H} \mathbf{x}_{k+1}+\mathbf{w}_{k+1}
\end{aligned}
$$

and the controllability matrix for such a plant is

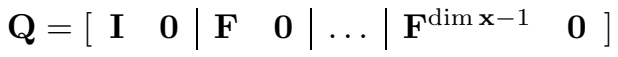

Consequently, the dimensionality of the controllable subspace, spanned by the column space of $\mathbf{Q},(\operatorname{Im} \mathbf{Q})$, is $\operatorname{rank} \mathbf{Q}=$ $\operatorname{dim} \mathbf{x}_{r}$, regardless of the number of landmarks in the map. Obviously, the only controllable states are the ones associated with the vehicle motion.

The observability matrix of our system becomes

$$
\mathbf{R}=\left[\begin{array}{c}
\mathbf{H} \\
\mathbf{H F} \\
\vdots \\
\mathbf{H F}^{\operatorname{dim} x-1}
\end{array}\right]
$$

The rank of $\mathbf{R}$ indicates the dimensionality of the observable subspace, which in turn, is spanned by the row space of $\mathbf{R}$, $\left(\operatorname{Im} \mathbf{R}^{\top}\right)$. rank $\mathbf{R}=\operatorname{dim} \mathbf{x}-\operatorname{dim} \mathbf{x}_{f^{(i)}}$.

\section{THE MONOBOT}

We return our attention now to the monobot. Consider the even more restrictive case in which only one landmark is available. By substituting the resulting expressions for the model Jacobians, the controllability and observability matrices reduce to

$$
\mathbf{Q}=\left[\begin{array}{ll}
1 & 1 \\
0 & 0
\end{array}\right], \quad \mathbf{R}=\left[\begin{array}{ll}
-1 & 1 \\
-1 & 1
\end{array}\right]
$$

The controllable subspace has a basis of the form $[q, 0]^{\top}$, clearly indicating that the only dimension in the state space that can be controlled is the one associated with the motion of the robot.

The observable subspace on the other hand, with basis $[r,-r]^{\top}$, shows how the observed robot and landmark locations are fully correlated. The unobservable subspace is the orthogonal complement of $\operatorname{Im} \mathbf{R}^{\top}$, and has a basis $[r, r]^{\top}$. An expression for it was already derived from the analysis of the total Fisher information matrix and is given in (6). The name $\mathbf{E}_{\mathrm{Ker} \mathbf{R}}$ indicates that it is a basis for the null space of $\mathbf{R}$.

A measure of the error incurred while trying to reconstruct the state $\tilde{\mathbf{x}}_{r}$ from correlated observations is given by the angle between these two subspaces. For the one landmark monobot, the angle is $\alpha=\angle \operatorname{Im} \mathbf{Q} \operatorname{Im} \mathbf{R}^{\top}=\pi / 4 \mathrm{rad}$.

There is one direction of the state space which is not observed, the one orthogonal to $\operatorname{Im} \mathbf{R}^{\top}$ (along $\operatorname{Ker} \mathbf{R}$ ). The information for the revision of $\tilde{\mathbf{x}}_{r}$ and $\tilde{\mathbf{x}}_{f}$ along the direction orthogonal to $\operatorname{Im} \mathbf{R}^{\top}$ is missing. The angle $\alpha$ indicates how close noise driven observations are from fully revising the robot part of the state space.

What happens if we add more landmarks to the environment? will the vehicle and landmark location estimates improve or degrade? will we be able to achieve an uncoupled reconstruction of the entire state space? The answer to the above questions is "improve" but "no".

Consider the two-landmark monobot case. A possible set of bases for the controllable and observable subspaces are

$$
\mathbf{E}_{\operatorname{Im} \mathbf{Q}}=\left(\begin{array}{l}
1 \\
0 \\
0
\end{array}\right), \quad \mathbf{E}_{\operatorname{Im} \mathbf{R}^{\top}}=\left(\begin{array}{rr}
1 & 1 \\
-1 & 0 \\
0 & -1
\end{array}\right)
$$




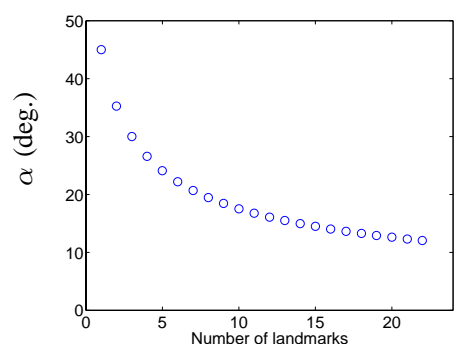

a)

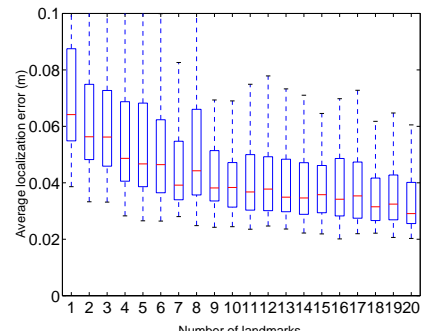

b)
Fig. 3. a) $\angle \mathcal{R}(\mathbf{Q}) \mathcal{R}\left(\mathbf{R}^{\top}\right)$. Angle between the observable and controllable subspaces. b) Reduction of the average monobot localization error $\mathbf{x}_{r, k}-$ $\mathbf{x}_{r, k \mid k}$ with respect to the number of landmarks used. The results correspond to a Montecarlo simulation over 100 SLAM runs. The dotted lines show the extent of the data for the entire set of runs, and the boxes contain marks at the lower, median and upper quartile

and the angle between these two subspaces can be computed as the smallest non null singular value of the product of their orthonormal bases [8]. $\alpha=\angle \operatorname{Im} \mathbf{Q} \operatorname{Im} \mathbf{R}^{\top}=163 \pi / 832 \mathrm{rad}$. Following this procedure we computed the value of $\alpha$ for a three-landmark monobot model, further reducing to $\alpha=$ $\pi / 6$. And, as we add more landmarks to the map, the angle between the observable and controllable subspaces reduces monotonically. Fig. 3a shows experimentally the decrease in $\alpha$ as landmarks are added to the map state model. Such monotonic reduction in $\alpha$ suggests that our measurement noise driven corrections to the map state estimate would reconstruct the vehicle localization estimate closer to the actual value of the vehicle pose.

Theorem 1 (proof in [5]): In the case of a linear onedimensional robot, the angle between the controllable and observable subspaces in the KF-SLAM algorithm depends only on the total number of landmarks used, $n$, and is given by $\alpha=\arccos \sqrt{\frac{n}{n+1}}$.

As the number of landmarks grows, the observable subspace gets closer to the controllable part of the state space (the vehicle localization states). $\lim _{n \rightarrow \infty} \alpha=0$.

It is unrealistic however, to have an infinite number of landmarks, and a compromise has to be made between the possibility of including as many landmarks as possible, and the amount of information that new observations give. Also one has to bear in mind that as we add more and more landmarks to the map, we will also introduce their associated measurement noise.

It has been argued that the performance of any SLAM algorithm would be enhanced by concentrating on fewer, better landmark observations [3]. That is certainly true, little gain (little reduction in $\alpha$ ) is attained when going from 25 to 125 landmarks compared to the move from 1 to 5 or 5 to 25 .

In Fig. 4 we have plotted the results of using the original fully correlated KF approach to SLAM for a monobot that starts at location $\mathbf{x}_{r, 0 \mid 0}=-1 m$, and moves along a straight line with a temporal sinusoid trajectory returning to the same point after 100 iterations. Landmarks are located at $\mathbf{x}_{f^{(i)}}=$
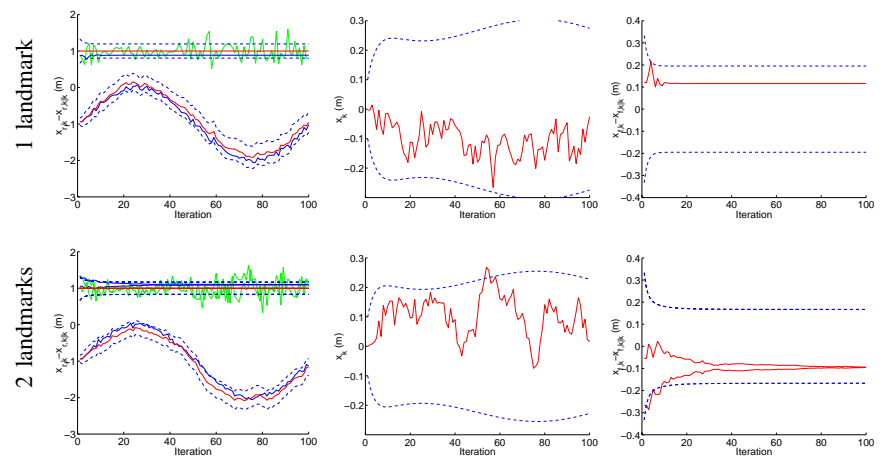

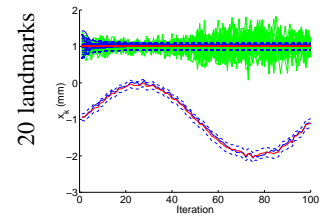

Robot and landmark localization

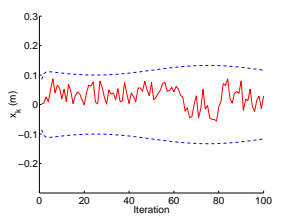

Vehicle error

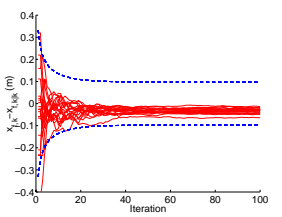

Landmark localization error
Fig. 4. Full-covariance KF SLAM for a monobot in a sinusoidal path from $\mathbf{x}_{r, 0 \mid 0}=-1 m$ to $\mathbf{x}_{100}=-1 m$ with 100 iterations. The noise corrupted sinusoidal vehicle trajectory is indicated by the darkest curve in the first column of plots. In the same set of figures, and close to it is a lighter curve that shows the vehicle location estimate as computed by the filter, along with a pair of dotted lines indicating $2 \sigma$ bounds on such estimate. The dark straight lines at the $1 \mathrm{~m}$ level indicate the landmark location estimates; and the lighter noise corrupted signals represent sensor measurements. Also shown, are a pair of dotted lines for $2 \sigma$ bounds on the landmark location estimates. The second column of plots shows the vehicle localization error and its corresponding covariance, also on the form of $2 \sigma$ dotted bounds. And, the last column shows the same for the landmark estimates.

$1 \mathrm{~m}$. A plant noise model proportional to the motion command, and a measurement noise model proportional to the distance from the sensor to the landmark are used. The dotted lines indicate $2 \sigma$ bounds on the state estimates.

The effects of partial observability manifest the dependence on the initial conditions. Note how both the vehicle and landmark mean localization errors do not converge to zero. Their steady state value is subject to the error incurred at the first observation. That is, the filter is marginally stable (the matrix $\mathbf{F}-\mathbf{K H F}$ has a pole in one [9]).

A Montecarlo simulation over 100 SLAM runs showed however filter unbiasedness, a property of optimal stochastic state estimation (Kalman filter). That is, the average landmark localization error over the entire set of simulations was still zero, thanks to the independence of the initial landmark measurement errors at each test run.

The steady state error for the robot and landmark localization is less sensitive to the initial conditions when a large number of landmarks are used. The reason is the same as for the Montecarlo simulation, the observations are independent, and their contribution averages at each iteration in the computation of the localization estimate. The results of the Montecarlo simulation are shown in Fig. 3b depicting the effect of the increase in the number of landmarks on the average vehicle localization error. 


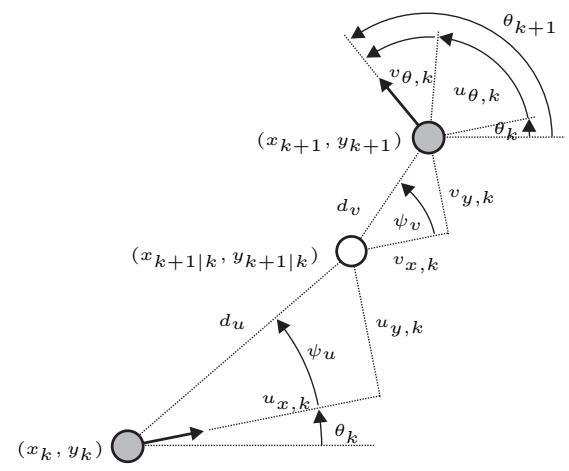

Fig. 5. Two-dimensional mobile robot motion model.

\section{THE PLANAR ROBOT}

The reconstructibility issues presented for the linear and one-dimensional robot of the previous section, nicely extend when studying more complicated platforms. Consider the planar robot shown in Fig. 5, a nonlinear wheeled vehicle with three degrees of freedom, and an environment consisting of two-dimensional point landmarks located on the floor.

The dimensionality of the controllable subspace is $\operatorname{dim} \mathbf{x}_{r}=$ 3 , and for the specific case in which only one landmark is available, a basis for the controllable subspace is simply

$$
\mathbf{E}_{\operatorname{Im} \mathbf{Q}}=\left(\begin{array}{c}
\mathbf{I} \\
\mathbf{0}_{2 \times 3}
\end{array}\right)
$$

The dimensionality of the observable subspace is, for this particular configuration, rank $\mathbf{R}=3$. This last result is easily verified with simple symbolic manipulation of the specific expression for the state model in [5]. Possible bases for $\operatorname{Im} \mathbf{R}^{\top}$, and for the null space of $\mathbf{R}$ (the unobservable subspace) are

$$
\mathbf{E}_{\mathrm{Im} \mathbf{R}^{\top}}=\left(\begin{array}{rrr}
1 & 0 & 0 \\
0 & 1 & 0 \\
0 & 0 & 1 \\
-1 & 0 & 0 \\
0 & -1 & 0
\end{array}\right) \quad \mathbf{E}_{\mathrm{Ker} \mathbf{R}}=\left(\begin{array}{ll}
1 & 0 \\
0 & 1 \\
0 & 0 \\
1 & 0 \\
0 & 1
\end{array}\right)
$$

The only independently observable state is the one associated to the robot orientation $\theta$. The other four states, the Cartesian coordinates of the robot and landmark locations span a space of dimension 2. Even when $\operatorname{Im} \mathbf{Q}$ and $\operatorname{Im} \mathbf{R}^{\top}$ both span $\mathbb{R}^{3}$, we see that the inequality $\operatorname{Im} \mathbf{Q} \neq \operatorname{Im} \mathbf{R}^{\top}$ still holds, as in the case of the monobot. That is, the observable and controllable subspaces for the one-landmark 3dof-robot SLAM problem correspond to different three-dimensional subspaces in $\mathbb{R}^{5}$; and, their intersection represents the only fully controllable and observable state, i.e., the robot orientation. Once more, a measure of the reconstruction error incurred when estimating the vehicle pose from correlated observations is given by the angle between these two subspaces.

Resorting again to a singular value decomposition for the computation of a pair of orthonormal bases for $\operatorname{Im} \mathbf{Q}$ and $\operatorname{Im} \mathbf{R}^{\top}$, we have that for the one-landmark planar robot case, $\alpha=\pi / 4 \mathrm{rad}$. For a two-landmark map, $\alpha=163 \pi / 832 \mathrm{rad}$, for a three-landmark model, $\alpha=\pi / 6$, and as we add more and more landmarks to the environment, the angle between the controllable and observable subspaces reduces monotonically, in exactly the same manner as in the case of the monobot.

Theorem 2 (proof also in [5]): In the case of a nonlinear planar robot with 3 degrees of freedom, the angle between the controllable and observable subspaces in the EKF-SLAM algorithm depends only on the total number of landmarks used, $n$, and is given by $\alpha=\arccos \sqrt{\frac{n}{n+1}}$.

\section{COMPlete OBSERVABILITy}

In Section III we characterized the unobservable subspace in SLAM as the subspace spanned by the null eigenvectors of the total Fisher information matrix. Furthermore, we showed in Sections IV-VI how the unobservable part of the state space is precisely a linear combination of the landmark and robot pose estimates.

In order to gain full observability we propose to extend the measurement model doing away with the constraint imposed by full correlation. We present two techniques to achieve this. One is to let one landmark serve as a fixed global reference, with its localization uncertainty independent of the vehicle pose.

The second proposed technique is the addition of a fixed external sensor, such as a camera, a GPS, or a compass, that can measure all or part of the vehicle location state at all times, independent of the landmark estimates.

Both techniques are based essentially on the same principle. Full observability requires an uncorrelated measurement Jacobian, or equivalently, a full rank Fisher information matrix.

\section{A. A fixed global reference}

The plant model is left untouched, i.e.,

$$
\mathbf{x}_{k+1}=\mathbf{x}_{k}+\mathbf{u}_{k}+\mathbf{v}_{k}
$$

The measurement model takes now the form

$$
\left[\begin{array}{c}
z_{k}^{(0)} \\
\mathbf{z}_{k}
\end{array}\right]=\left[\begin{array}{cc}
-1 & \mathbf{0}_{1 \times n} \\
-\mathbf{1}_{n \times 1} & \mathbf{I}
\end{array}\right] \mathbf{x}+\left[\begin{array}{c}
w_{k}^{(0)} \\
\mathbf{w}_{k}
\end{array}\right]
$$

One of the observed landmarks is to be taken as a global reference at the world origin. No map state is needed for it. The zero-th superscript in the measurement vector is used for the consistent indexing of landmarks and observations with respect to the original model. It can be easily shown that the observability matrix for this new model is full rank.

The innovation covariance matrix for the augmented system $\mathbf{S}_{O, k}$ is of size $(n+1) \times(n+1)$, and its inverse can be decomposed in

$$
\mathbf{S}_{O, k}^{-1}=\left[\begin{array}{cc}
\varsigma_{O, 00} & \varsigma_{O, 01} \cdots \varsigma_{O, 0 n} \\
\vdots & \hat{\mathbf{S}}_{k}^{-1} \\
\varsigma_{O, 0 n} &
\end{array}\right]
$$

with $\varsigma_{O, i j}$ the $i j$-th entry in $\mathbf{S}_{O, k}^{-1}, \varsigma_{O}=$ $\left[\sum \varsigma_{O, 1 i}, \ldots, \sum \varsigma_{O, 1 i}\right]$, and $\hat{\mathbf{S}}_{k}^{-1}$ its submatrix associated to the landmarks that are under estimation (excluding the anchor observation). 
The $k$-th element of the Fisher information matrix sum in (3) is now

$$
\mathbf{J}_{O, k}=\left[\begin{array}{cc}
\sum \sum \varsigma_{O, i j} & -\varsigma_{O} \\
-\boldsymbol{\varsigma}_{O}^{\top} & \hat{\mathbf{s}}_{k}^{-1}
\end{array}\right]
$$

Unlike in (4), this form of the Fisher information matrix is full rank. Moreover, from the properties of positive definite matrices, if $\mathbf{J}_{O, k}$ is positive definite, the entire sum that builds up $\mathbf{J}_{O}$ is also positive definite.

Fig. 6 shows the results of applying full observability to the same monobot model as the one portrayed in Fig. 4. Note how the steady state (robot pose and landmark locations) is now unbiased with respect to the initial state estimates. State covariances are also smaller than those in Fig. 4.

\section{B. An external sensor}

Instead of using one of the landmarks as a global reference, one could also use a fixed sensor to measure the position of the robot. For example, by positioning a camera that observes the vehicle at all times. For such cases, the monobot measurement model may take the form

$$
\left[\begin{array}{c}
z_{k}^{(0)} \\
\mathbf{z}_{k}
\end{array}\right]=\left[\begin{array}{cc}
1 & \mathbf{0}_{1 \times n} \\
-\mathbf{1}_{n \times 1} & \mathbf{I}
\end{array}\right] \mathbf{x}+\left[\begin{array}{c}
w_{k}^{(0)} \\
\mathbf{w}_{k}
\end{array}\right]
$$

The characteristics of the observability matrix, and the Fisher information matrix, are exactly the same as for the previous case. This new model is once more, fully observable. The results are theoretically equivalent to the previous case. The choice of one technique over the other would depend on the availability of such external sensor, and on its measurement noise covariance characteristics.

The key point here is that we have proved that full observability, i.e., zero mean state convergence, is indeed possible in SLAM without the need of an oracle (an external sensor), but by simply anchoring the first observed landmark to the global reference frame.

\section{Planar vehicle}

The results from the previous section are easily extensible to more complicated vehicle models, provided the linearization technique chosen is sufficiently accurate. For example, the measurement model of a global reference fixed at the origin, for the nonlinear vehicle from Fig. 5 is

$$
\mathbf{h}^{(0)}=-\mathbf{R}^{\top} \mathbf{t}+\mathbf{w}^{(0)}
$$

and its corresponding Jacobian is

$$
\mathbf{H}_{\mathbf{x}}^{(0)}=\left[\begin{array}{lll}
-\mathbf{R}^{\top} & -\dot{\mathbf{R}}^{\top} \mathbf{t} & \mathbf{0}_{2 \times 2 n}
\end{array}\right]
$$

The case of the external sensor is even simpler, the corresponding equations are

$$
\begin{aligned}
\mathbf{h}^{(0)} & =\mathbf{t}+\mathbf{w}^{(0)} \\
\mathbf{H}_{\mathbf{x}}^{(0)} & =\left[\begin{array}{ll}
\mathbf{I} & \mathbf{0}_{2 \times(2 n+1)}
\end{array}\right]
\end{aligned}
$$

In both cases, the symbolic manipulation of (18) and (20) with a commercial algebra package, produced full rank observability matrices. That is, for the planar mobile robot platform used, only one two-dimensional global reference, or the use of a sensor that can measure the $x y$ position of the robot, are sufficient to attain full observability in SLAM.
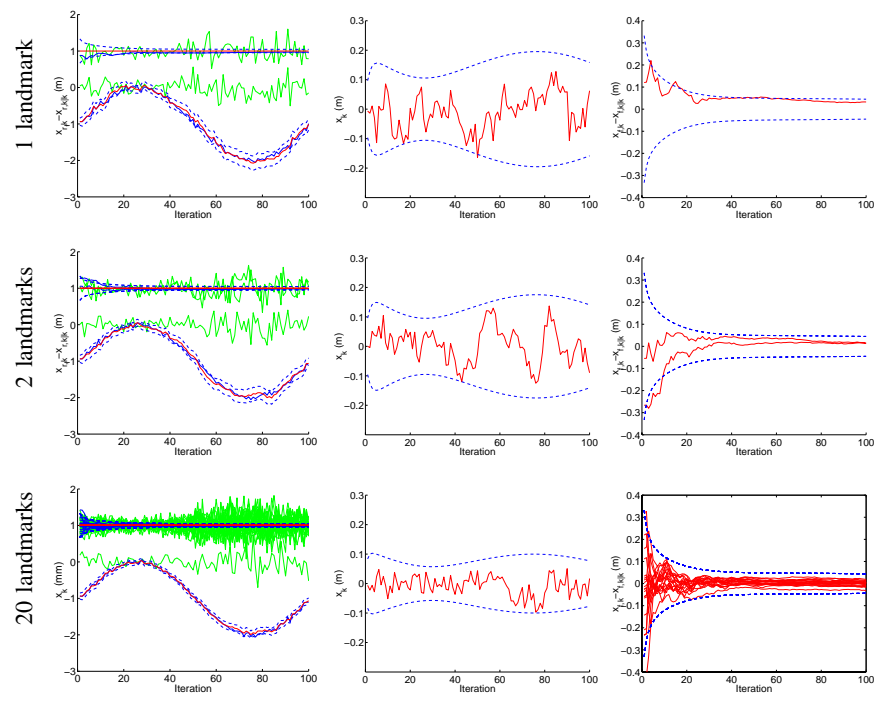

Robot and landmark localization

Vehicle error

Landmark localization error

Fig. 6. Full-covariance fully observable KF SLAM for a monobot in a sinusoidal path from $\mathbf{x}_{r, 0 \mid 0}=-1 m$ to $\mathbf{x}_{100}=-1 m$ with 100 iterations. The global reference is observed at the origin.

\section{CONCLUSION}

We have shown how full correlation of the map model in KF-SLAM hinders full observability of the state estimate. Partial observability makes the final map dependant on the initial observations, and does not guarantee convergence to a positive definite covariance matrix. This situation can easily be remedied either by anchoring the map to the first landmark observed, or by having an external sensor that sees the vehicle at all times.

\section{REFERENCES}

[1] R. C. Smith and P. Cheeseman, "On the representation and estimation of spatial uncertainty," Int. J. Robot. Res., vol. 5, no. 4, pp. 56-68, 1986.

[2] M. W. M. G. Dissanayake, P. Newman, S. Clark, H. F. Durrant-Whyte, and M. Csorba, "A solution to the simultaneous localization and map building (SLAM) problem," IEEE Trans. Robot. Automat., vol. 17, no. 3, pp. 229-241, Jun. 2001.

[3] P. W. Gibbens, G. M. W. M. Dissanayake, and H. F. Durrant-Whyte, "A closed form solution to the single degree of freedom simultaneous localisation and map building (SLAM) problem," in Proc. IEEE Int. Conf. Decision Control, Sydney, Dec. 2000, pp. 408-415.

[4] Y. Bar-Shalom, X. R. Li, and T. Kirubarajan, Estimation with Applications to Tracking and Navigation. New York: John Wiley \& Sons, 2001.

[5] J. Andrade-Cetto, "Environment learning for indoor mobile robots," Ph.D. dissertation, UPC, Barcelona, Apr. 2003.

[6] R. Todling, "Estimation theory and foundations on atmosferic data assimilation," Data Assimilation Office. Goddard Space Flight Center, Tech. Rep. DAO Note 1999-01, 1999.

[7] J. Neira and J. D. Tardós, "Data association in stochastic mapping using the joint compatibility test," IEEE Trans. Robot. Automat., vol. 17, no. 6, pp. 890-897, Dec. 2001.

[8] I. B. Risteski and K. G. Trenčevski, "Principal values and principal subspaces of vector spaces with inner product," Cont. Algebra Geom., vol. 42, no. 1, pp. 289-300, 2001.

[9] T. Vidal-Calleja, J. Andrade-Cetto, and A. Sanfeliu, "Estimator stability analysis in SLAM," IRI, UPC, Tech. Rep. IRI-DT-04-01, Feb. 2004, submitted to 5th Symposium on Intelligent Autonomous Vehicles. 